\title{
Diversity Combining in Hybrid RF/FSO Systems with PSK Modulation
}

\author{
Nestor D. Chatzidiamantis*, George K. Karagiannidis*, Emmanouil E. Kriezis* and Michail Matthaiou ${ }^{\dagger}$ \\ *Department of Electrical \& Computer Engineering, Aristotle University of Thessaloniki, Greece, Emails: \{nestoras, geokarag, mkriezis\}@auth.gr \\ ${ }^{\dagger}$ Department of Signals and Systems, Chalmers University of Technology, Sweden, Email: michail.matthaiou@chalmers.se
}

\begin{abstract}
We present a novel architecture for hybrid radio frequency (RF)/ free space optical (FSO) wireless systems without feedback or channel state information (CSI) at the transmitter. Under the assumption that $60 \mathrm{GHz}$ RF and FSO systems support the same data rates, the proposed implementation transmits the same data over both links, using phase shift keying (PSK) as a common modulation scheme, and combines the signals from each individual link at the receiver on a symbol-bysymbol basis. Two popular diversity combining schemes are considered, namely, selection combining (SC) and maximal ratio combining (MRC), while tractable analytical approximations for the bit error rate (BER) are obtained. Investigations over various weather conditions and link distances revealed that the proposed implementation fully exploits the complementary nature of RF and FSO channels, even when one of the two available links fails. Furthermore, the comparison of the combining schemes demonstrates MRC as the optimum combining scheme, offering link distance gains compared to SC.
\end{abstract}

\section{INTRODUCTION}

The constant need for higher data rates in support of the demanding applications has led to the development of the Free Space Optical (FSO) and millimeter wavelength (MMW) radio frequency $(\mathrm{RF})$ communication technologies. Operating at unlicensed optical and $60 \mathrm{GHz}$ frequencies, FSO and MMW RF systems offer the potential of broadband capacity (up to a few Gbps) in low cost and over distances of up to a few kilometers [1], [2]. Despite their common features, FSO and MMW RF systems are not affected in the same way by atmospheric and weather effects. Several studies [3]- [4] have shown that fog is the main degrading factor in FSO links, while rain does not cause significant effects. On the contrary, $60 \mathrm{GHz}$ RF links are susceptible to heavy rain conditions and oxygen absorption [2]- [4], while fog has no particular effect.

This complementary behavior of RF and FSO channels has led to various proposals of hybrid RF/FSO implementations. In [5], a coding scheme for hybrid RF/FSO systems was suggested, which adjusts its code rate (in RF and FSO channels) based on the instantaneous channel state information (CSI). Despite its efficiency, this scheme requires instantaneous CSI at the transmitter, which makes its implementation sophisticated. As a consequence, more practical coding schemes were proposed. In [6], a hybrid RF/FSO system with bit-interleaved coded modulation was presented, which offers significant performance gains; however, it requires statistical CSI, i.e., knowledge of the hybrid channel's statistical parameters, in its code design at the transmitter. Furthermore, in [7] and [8], an efficient coding scheme based on Raptor codes was designed and implemented; however, the need for providing feedback to transmitter remains. To avoid the employment of a feedback channel, the commercial implementation of [9] was proposed. In this scheme, the transmitter sends the same data over both links and the receiver, after detecting the received data stream at each link independently, selects the data stream that is deemed more reliable. Despite its robustness against instantaneous interruptions, this scheme combines the received signals only on a frame-by-frame basis, without allowing popular diversity combining methods [10] to be applied in order to improve the system's overall performance.

In this paper, we introduce an alternative hybrid RF/FSO implementation for the case when there is no feedback or CSI at the transmitter. Under the assumption that FSO and MMW RF systems support the same data rates, the proposed system transmits the same information over both links using the same type of modulation as well. Phase shift keying is the modulation scheme which is employed in both channels, since it can be easily implemented not only in MMW RF systems [11], but also in FSO systems [12]. Hence, the signals which are received from each individual link at the receiver can be directly combined in the electrical domain, on a symbol-bysymbol basis. Two popular combining methods are investigated: selection combining (SC) that processes only the output of the link with the highest electrical signal-to-noise ratio (SNR), and maximal ratio combining (MRC) that processes the outputs of both links maximizing the overall electrical SNR. For these reception methods, analytical approximations for the bit error rate (BER) are derived and investigations over various weather conditions and link distances are carried out.

The remainder of the paper is organized as follows. In Section II, the proposed system model is described. In Section III, the suggested combining methods at the receiver are outlined and analytical expressions for their BER performance are obtained. Section IV investigates the performance of the combining methods under various weather conditions and link distances, and finally, useful concluding remarks are summarized in Section V.

Notation: Before continuing, some notational comments are in order: $\mathbb{E}\{\cdot\}$ denotes statistical expectation, $K_{\nu}(\cdot)$ is the $\nu$ th order modified Bessel function of the second kind [13, Eq. (8.407)], $\Gamma(\cdot)$ and $\Gamma(\cdot, \cdot)$ are the Gamma [13, Eq. (8.310)] and the upper incomplete Gamma [13, Eq. (8.350/2)] functions, respectively. Furthermore, $I_{n}(\cdot)$ is $n$-th order modified Bessel function of the first kind [13, Eq. (8.406)], $G_{p, q}^{m, n}[\cdot]$ is the 
Meijer's $G$-function [13, Eq. (9.301)], $Q(\cdot)$ is the Gaussian Q-function, $\operatorname{erfc}(\cdot)$ is the complementary error function and $Q_{1}(\cdot, \cdot)$ is the Marcum function [10, Eq. (4.34)].

\section{SySTEM MOdEL}

At the transmitter, information is modulated using PSK and the output of the modulator is transmitted through both FSO and RF links. The PSK modulated signal, which is used as an input to the available sub-systems, can be expressed as

$$
x(t)=\sum_{k} g(t-k T) \cos \left(2 \pi f_{s} t+\phi_{k}\right)
$$

where $T$ denotes the symbol period, $f_{s}$ is the frequency of the PSK subcarrier which must satisfy $f_{s}=\frac{q}{T}$ with $q \geq 1, g(t)$ is the shaping pulse, $\phi_{k} \in\left[0, \ldots,(M-1) \frac{2 \pi}{M}\right]$ is the phase of the $k$ th transmitted symbol and $M$ is the modulation order, which depends upon the bit transmission rate, $R_{b}$, according to $R_{b}=$ $\frac{\log _{2} M}{T}$. Next, we present the equivalent discrete time received FSO and RF signals of the proposed hybrid communication system.

\section{A. Transmission through the FSO sub-system}

1) Signal Model: We consider an FSO sub-system with intensity modulation and direct detection (IM/DD). The PSK signal can be directly modulated on the transmitter's laser intensity; however, a DC bias must be added in order to ensure that the transmitted PSK waveform always satisfies the non-negativity input constraint. Hence, the intensity of the transmitted optical signal is written as

$$
I(t)=P_{1}[1+\mu x(t)]
$$

where $P_{1}$ is the average transmitted optical power and $\mu$ is the modulation index $(0<\mu<1)$ that ensures that the laser avoids over-modulation induced clipping.

At the receiver end of the FSO sub-system, the optical power which is incident on the photodetector is converted into an electrical signal through direct detection. We assume operation in the high SNR regime where the shot noise caused by ambient light is dominant and therefore the Gaussian noise model is used as a good approximation of the Poisson photon counting detection model.

After demodulating the electrical output of the photodetector, the DC bias is filtered out and the received discrete-time equivalent signal, which is obtained at the output of the FSO receiver, is modeled as

$$
r_{1}[k]=\mu \eta \sqrt{\frac{E_{g}}{2}} P_{1} h_{1} g_{1} x[k]+n_{1}[k]
$$

where $\eta$ corresponds to the receiver's optical-to-electrical efficiency, $x[k]=\cos \phi_{k}+j \sin \phi_{k}, E_{g}$ is the energy of the shaping pulse and $n_{1}[k]$ is the signal-independent zero mean circularly symmetric complex Gaussian noise component with $\mathbb{E}\left\{n_{1}[k] n_{1}^{*}[k]\right\}=2 \sigma_{1}^{2}=\sigma_{n_{1}}^{2}$. Furthermore, $g_{1}$ and $h_{1}$ represent the average gain of the FSO link and the turbulenceinduced fading coefficient with $\mathbb{E}\left\{h_{1}\right\}=1$. Assuming perfect alignment between the transmitter and receiver, the attenuation factor $g_{1}$ is provided by [9]

$$
g_{1}=\frac{\pi D^{2}}{4(\theta L)^{2}} \exp \left(-\alpha_{1} L\right)
$$

where $\alpha_{1}$ denotes the weather dependent attenuation coefficient (in $1 / \mathrm{km}$ ), $\theta$ is the transmit beam divergence, $D$ is receiver's aperture diameter and $L$ is the link distance. For the statistical parameter $h_{1}$, the well-known Gamma-Gamma fading model is adopted with probability density function (pdf) provided by [14, Eq. (60)-(61)]

$$
f_{1}\left(h_{1}\right)=\frac{2(\alpha \beta)^{\frac{\alpha+\beta}{2}}}{\Gamma(\alpha) \Gamma(\beta)} h_{1}^{\frac{\alpha+\beta}{2}-1} K_{\alpha-\beta}\left(2 \sqrt{\alpha \beta h_{1}}\right),
$$

where $h_{1} \geq 0$ and the parameters $\alpha>0$ and $\beta>0$ are calculated based on the link distance, $L$, the diameter of the receiver aperture, $D$, the weather dependent index of refraction, $C_{n}^{2}$, and the wavelength of the optical carrier, $\lambda_{1}$, using [14, Eq.s (60)-(61)].

2) SNR Statistics: Based on (3), the instantaneous electrical SNR at the output of the FSO receiver can be defined as

$$
\gamma_{1}=\bar{\gamma}_{1} h_{1}^{2}
$$

where $\bar{\gamma}_{1}$ is the average electrical SNR given by

$$
\bar{\gamma}_{1}=\frac{\mu^{2} \eta^{2} P_{1}^{2} E_{s} g_{1}^{2}}{\sigma_{n_{1}}^{2}}
$$

with $E_{s}=\frac{E_{g}}{2}$. After a simple transformation of the random variable (rv) $h_{1}$, the pdf of the electrical SNR, $\gamma_{1}$, is derived as

$$
f_{\gamma_{1}}\left(\gamma_{1}\right)=\frac{(\alpha \beta)^{\frac{\alpha+\beta}{2}}}{\Gamma(\alpha) \Gamma(\beta) \bar{\gamma}_{1}^{\frac{\alpha+\beta}{4}}} \gamma_{1}^{\frac{\alpha+\beta}{4}-1} K_{\alpha-\beta}\left(2 \sqrt{\alpha \beta \sqrt{\frac{\gamma_{1}}{\bar{\gamma}_{1}}}}\right)
$$

while its cumulative density function (cdf) can be expressed as

$$
F_{\gamma_{1}}\left(\gamma_{1, t h}\right)=\frac{1}{\Gamma(\alpha) \Gamma(\beta)} G_{1,3}^{2,1}\left[\alpha \beta \sqrt{\frac{\gamma_{1, t h}}{\bar{\gamma}_{1}}} \mid \begin{array}{c}
1 \\
\alpha, \beta, 0
\end{array}\right] .
$$

\section{B. Transmission through the MMW RF sub-system}

1) Signal Model: At the transmitter of the RF sub-system, the PSK-modulated signal is upconverted to MMW RF carrier frequency and transmitted through the RF link. The received RF signal is demodulated and the discrete time output of the $\mathrm{RF}$ receiver is modeled as

$$
r_{2}[k]=\sqrt{P_{2}} \sqrt{g_{2}} h_{2} \sqrt{E_{s}} x[k]+n_{2}[k]
$$

where $P_{2}$ represents the RF transmit power, $g_{2}$ is the average power gain of the RF link, $h_{2}$ is the RF fading gain with $\mathbb{E}\left\{h_{2}^{2}\right\}=1, x[k]$ is taken from the $M$-ary PSK alphabet, $E_{s}$ is defined earlier and $n_{2}[k]$ is the zero mean circularly symmetric complex Gaussian noise component with $\mathbb{E}\left\{n_{2}[k] n_{2}^{*}[k]\right\}=\sigma_{n_{2}}^{2}$. Assuming a carrier frequency of 60 
$\mathrm{GHz}$, the average power gain of the RF link is given by [6, Eq. (7)]

$$
g_{2}[d B]=G_{t}+G_{R}-20 \log _{10}\left(\frac{4 \pi L}{\lambda_{2}}\right)-\alpha_{\text {oxy }} L-\alpha_{\text {rain }} L,
$$

where $G_{t}$ and $G_{i}$ denote the transmit and receive antenna gains, respectively, $\lambda_{2}$ is the wavelength of the RF system, and $\alpha_{o x y}$ and $\alpha_{\text {rain }}$ are the attenuations caused by oxygen absorbtion and rain, respectively. The noise variance in the RF link is given by [6] $\sigma_{n_{2}}^{2}[d B m]=B+N_{o}+N_{F}$, where $B$ is the RF bandwidth (in dBMHz), $N_{o}$ is the noise power spectral density (in $\mathrm{dBm} / \mathrm{MHz}$ ) and $N_{F}$ is the noise figure of the receiver. Further, the fading gain, $h_{2}$, can be modeled as Ricean distributed [6], with pdf provided by [10, pp. 21].

2) SNR Statistics: The instantaneous SNR of the RF link is defined as

$$
\gamma_{2}=\bar{\gamma}_{2} h_{2}^{2}
$$

with $\bar{\gamma}_{2}$ denoting the average SNR given by

$$
\bar{\gamma}_{2}=\frac{P_{2} g_{2} E_{s}}{\sigma_{n_{2}}^{2}} .
$$

According to [10, pp. 420], the pdf of $\gamma_{2}$ is given by

$$
\begin{aligned}
f_{\gamma_{2}}\left(\gamma_{2}\right)= & \frac{K+1}{\bar{\gamma}_{2}} \exp \left(-(K+1) \frac{\gamma_{2}}{\bar{\gamma}_{2}}-K\right) \\
& \times I_{0}\left(2 \sqrt{K(K+1) \frac{\gamma_{2}}{\bar{\gamma}_{2}}}\right)
\end{aligned}
$$

where $K$ is the Ricean factor that depends on various link parameters, such as link distance or antenna height. Furthermore, its cdf can be expressed as

$$
F_{\gamma_{2}}\left(\gamma_{2, t h}\right)=1-Q_{1}\left(\sqrt{2 K}, \sqrt{\frac{2(1+K)}{\bar{\gamma}_{2}} \gamma_{2, t h}}\right) .
$$

The specific values for the parameters of the respective FSO and RF channel models were taken from [6], [9] and are summarized in Tables I and II.

\section{HYBRID RF/FSO WITH DIVERSITY COMBINING}

In this section, we investigate means by which the output signals of the transmission sub-systems can be combined at the receiver in order to produce a signal that is resilient against the atmospheric effects.

\section{A. No Combining}

As a benchmark, we present the case where only one of the two links is available at the receiver. Since PSK modulation is employed, the conditioned on the fading coefficient BER performance of each available link is given by [15, Eq. $(5.2 .61)]$

$$
P_{i}\left(e \mid \gamma_{i}\right)=A Q\left(\sqrt{2 \gamma_{i}} B\right) \quad i=1,2
$$

or equivalently by

$$
P_{i}\left(e \mid \gamma_{i}\right)=\frac{A}{2} \operatorname{erfc}\left(\sqrt{\gamma_{i}} B\right), \quad i=1,2
$$

where $A=1, B=1$ when $M=2$ (BPSK), and $A=\frac{2}{\log _{2} M}$, $B=\sin \frac{\pi}{M}$ when $M>2$.

\begin{tabular}{|c|c|c|}
\hline \multicolumn{3}{|c|}{ PSK modulation } \\
\hline Parameter & Symbol & Value \\
\hline Symbol rate & $\frac{1}{T}$ & $250 \mathrm{Msym} / \mathrm{s}$ \\
\hline PSK subcarrier frequency & $f_{s}$ & $250 \mathrm{MHz}$ \\
\hline Bandwidth & $B$ & $250 \mathrm{MHz}$ \\
\hline Energy of shaping pulse & $E_{g}$ & $2 \mathrm{~J}$ \\
\hline \multicolumn{2}{|c|}{ FSO sub-system } \\
\hline Parameter & Symbol & Value \\
\hline Wavelength & $\lambda_{1}$ & $1.55 \mu \mathrm{m}$ \\
\hline Optical Power & $P_{1}$ & $40 \mathrm{~mW}$ \\
\hline modulation index & $\mu$ & 1 \\
\hline Receiver Diameter & $D$ & $20 \mathrm{~cm}$ \\
\hline divergence angle & $\theta$ & $10 \mathrm{mrad}$ \\
\hline Responsivity & $\eta$ & $0.5 \mathrm{~A}$ \\
\hline Noise Variance & $\sigma_{1}$ & $10^{-7} \mathrm{~A} / \mathrm{Hz}$ \\
\hline \multicolumn{2}{|c|}{ RF sub-system } \\
\hline Parameter & $S y m b o l$ & Value \\
\hline Carrier frequency & $f_{c}$ & $60 \mathrm{GHz}$ \\
\hline Transmit Power & $P_{2}$ & $10 \mathrm{~mW}$ \\
\hline Transmit Antenna Gain & $G_{i}$ & $44 \mathrm{dBi}$ \\
\hline Receive Antenna Gain & $G_{r}$ & $44 \mathrm{dBi}$ \\
\hline Attenuation (oxygen) & $\alpha_{o x y}$ & $15.1 \mathrm{~dB} / \mathrm{km}$ \\
\hline Ricean Factor & $K$ & $6 \mathrm{~dB}$ \\
\hline Noise Power Spectral Density & $N_{o}$ & $-114 \mathrm{dBm} / \mathrm{MHz}$ \\
\hline Receiver Noise Figure & $N_{F}$ & $5 \mathrm{~dB}$ \\
\hline \multicolumn{2}{|c|}{} \\
\hline Pon
\end{tabular}

TABLE I

PARAMETERS OF THE HYBRID SYSTEM [6], [9] AND [12].

\begin{tabular}{|c|c|c|c|}
\hline Weather conditions & $\alpha_{1}(\mathrm{~dB} / \mathrm{km})$ & $\alpha_{\text {rain }}(\mathrm{dB} / \mathrm{km})$ & $C_{n}^{2}$ \\
\hline \hline Clear air & 0.43 & 0 & $5 \cdot 10^{-14}$ \\
\hline Moderate Fog & 42.2 & 0 & $2 \cdot 10^{-15}$ \\
\hline Moderate rain $(12.5 \mathrm{~mm} / \mathrm{h})$ & 5.8 & 5.6 & $5 \cdot 10^{-15}$ \\
\hline
\end{tabular}

TABLE II

WEATHER DEPENDENT PARAMETERS OF FSO AND RF CHANNELS [6].

1) FSO Link: The average BER when only the FSO signal is available at the receiver, will be obtained by

$$
\bar{P}_{1}=\int_{0}^{\infty} P_{1}\left(e \mid \gamma_{1}\right) f_{\gamma_{1}}\left(\gamma_{1}\right) d \gamma_{1} .
$$

which can be evaluated by expressing its integrands in terms of Meijer's $G$-functions, according to [16, Eq. (8.4.14/2)] and [16, Eq. (8.4.23/1)], and using [16, Eq. (2.24.1/1)]. Hence, an analytical solution is obtained as follows

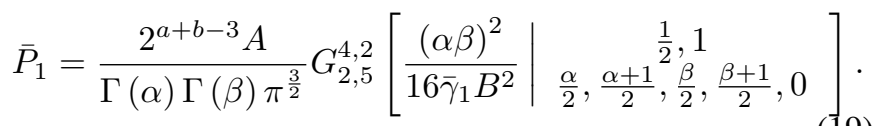

2) RF MMW Link: The average BER when only the RF MMW signal is available at the receiver, will be obtained by

$$
\bar{P}_{2}=\int_{0}^{\infty} P_{2}\left(e \mid \gamma_{2}\right) f_{\gamma_{2}}\left(\gamma_{2}\right) d \gamma_{2}
$$

By applying integration by parts and the transformation $x=$ $B \sqrt{2 \gamma_{2}}$, (20) simplifies to

$$
\bar{P}_{2}=\frac{A}{\sqrt{2 \pi}} \int_{0}^{\infty} \exp \left(-\frac{x^{2}}{2}\right) F_{\gamma_{2}}\left(\frac{x^{2}}{2 B^{2}}\right) d x,
$$


which can be analytically evaluated using (15) and [17, Eq. (62)]. Hence, a closed-form solution yields as

$$
\begin{aligned}
\bar{P}_{2} & =A Q_{1}\left(\sqrt{\frac{K}{2}}(1-q), \sqrt{\frac{K}{2}}(1+q)\right) \\
& -\frac{A(1+q)}{2} \exp \left(-\frac{K\left(1+q^{2}\right)}{2}\right) I_{0}\left(\frac{p K q^{2}}{2}\right)
\end{aligned}
$$

where $q=\frac{1}{\sqrt{1+p}}$ and $p=\frac{(1+K)}{\bar{\gamma}_{2} B^{2}}$.

\section{B. Selection Combining (SC)}

Perhaps the simplest combining method, SC measures the electrical SNR at each available link and selects the output signal of the sub-system with the highest SNR value. Hence the output SNR is given by

$$
\gamma_{S C}=\max \left(\gamma_{1}, \gamma_{2}\right) .
$$

The average BER of the SC receiver will be obtained by

$$
\bar{P}_{S C}=A \int_{0}^{\infty} Q\left(\sqrt{2 \gamma_{S C}} B\right) f_{\gamma_{S C}}\left(\gamma_{S C}\right) d \gamma_{S C}
$$

where $f_{\gamma_{S C}}(\cdot)$ is the pdf of $\gamma_{S C}$. Eq. (24) is equivalent with

$$
\bar{P}_{S C}(e)=\frac{A}{\sqrt{2 \pi}} \int_{0}^{\infty} \exp \left(-\frac{x^{2}}{2}\right) F_{\gamma_{S C}}\left(\frac{x^{2}}{2 B^{2}}\right) d x
$$

with $F_{\gamma_{S C}}(\cdot)$ denoting the cdf of $\gamma_{S C}$.

Due to the selection rule of (23), the cdf of $\gamma_{S C}$ is given by

$$
F_{\gamma_{S C}}(\gamma)=F_{\gamma_{1}}(\gamma) F_{\gamma_{2}}(\gamma)
$$

Proposition 1: The average BER of the SC receiver can be approximated by

$$
\bar{P}_{S C}=\bar{P}_{1}(e)-J-\varepsilon_{J}(N)
$$

where $\bar{P}_{1}(e)$ is the BER of the FSO syb-system evaluated by (19), $J$ is given by

$$
\begin{aligned}
J= & \frac{A \exp (-K) 2^{\alpha+\beta-2}}{\Gamma(\alpha) \Gamma(\beta) \pi^{\frac{3}{2}}} \\
& \times \sum_{i=0}^{N} \sum_{j=0}^{i} \frac{K^{i}(1+K)^{j} q^{2 j+1}}{\bar{\gamma}_{2}^{j} B^{2 j} 2^{j} i ! j !} \\
& \times G_{3,6}^{4,3}\left[\frac{\alpha^{2} \beta^{2} q^{2}}{16 \bar{\gamma}_{1} B^{2}} \mid \frac{\alpha}{2}, \frac{\alpha+1}{2}, \frac{\beta}{2}, \frac{\frac{\beta}{2}}{2}, \frac{\beta+1}{2}, 0, \frac{1}{2}\right]
\end{aligned}
$$

and $\varepsilon_{J}(N)$ represents an error term, upper bounded by

$$
\left|\varepsilon_{J}(N)\right| \leq\left(1-\frac{\Gamma(N+1, K)}{N !}\right) \bar{P}_{1}(e) .
$$

Proof: A detailed proof is given in Appendix I.

\section{Maximal Ratio Combining (MRC)}

In this diversity scheme, the received signals of each subsystem are combined in such a manner that the SNR of the signal at the output of the combiner is the sum of the SNRs of each sub-system. This can be achieved by applying the following combining rule

$$
r_{M R C}=\sqrt{\gamma_{1}} \frac{r_{1}}{\sigma_{n_{1}}}+\sqrt{\gamma_{2}} \frac{r_{2}}{\sigma_{n_{2}}},
$$

and, as a consequence, the output SNR will be obtained by

$$
\gamma_{M R C}=\gamma_{1}+\gamma_{2} .
$$

Proposition 2: The average BER performance of the MRC receiver can be approximated by

$$
\bar{P}_{M R C} \approx \frac{A}{12} M\left(B^{2}\right)+\frac{A}{4} M\left(\frac{4 B^{2}}{3}\right)
$$

where $M(\cdot)$ is the function defined as

$$
\begin{aligned}
M(x)= & \frac{2^{\alpha+\beta-2}(1+K) \exp \left(-\frac{K x \bar{\gamma}_{2}}{1+K+x \bar{\gamma}_{2}}\right)}{\Gamma(\alpha) \Gamma(\beta) \pi\left(1+K+x \bar{\gamma}_{2}\right)} \\
& \times G_{1,4}^{4,1}\left[\frac{(\alpha \beta)^{2}}{16 \bar{\gamma}_{1} x} \mid \frac{\alpha}{2}, \frac{\alpha+1}{2}, \frac{\beta}{2}, \frac{\beta+1}{2}\right] .
\end{aligned}
$$

Proof: A detailed proof is given in Appendix II.

\section{Results \& Discussion}

In this section, we present numerical results for the BER performance of the proposed hybrid RF/FSO system that employs either MRC or SC at the receiver, under various weather conditions. We further compare its performance with the benchmarking scheme of no combining, where only one link is available. The adopted values for the various system and channel parameters are obtained by Tables I and II.

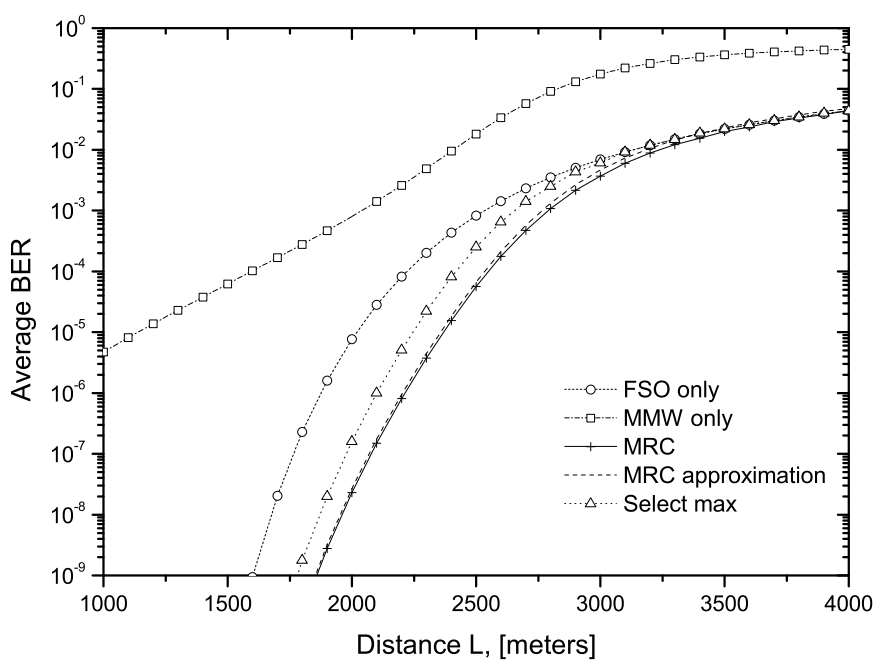

Fig. 1. BER performance of the hybrid RF/FSO system under consideration in clear weather conditions.

Figures 1-3 illustrate the average BER performance of the hybrid RF/FSO system under consideration, when $M=4$ 


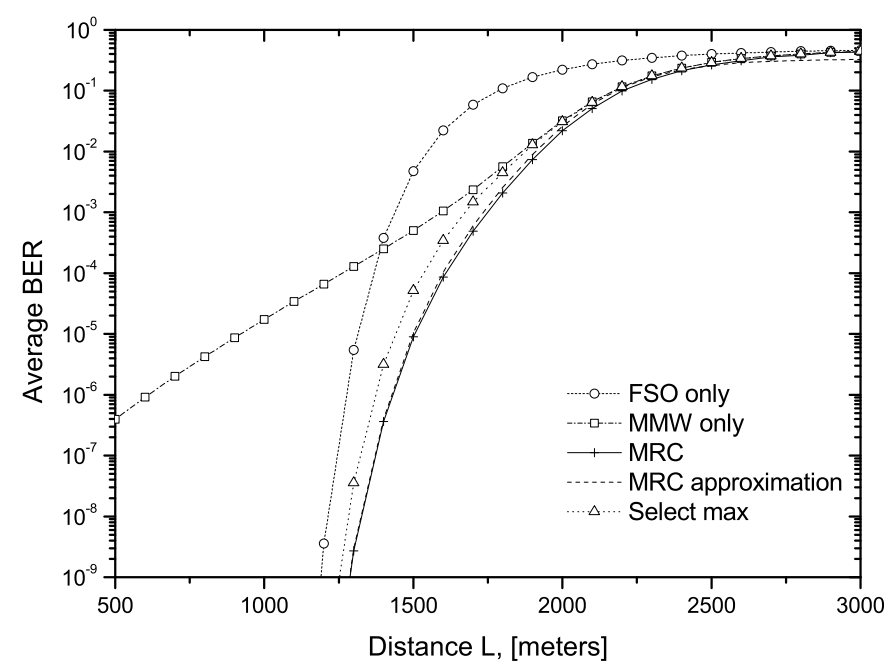

Fig. 2. BER performance of the hybrid RF/FSO system under consideration in moderate rain conditions.

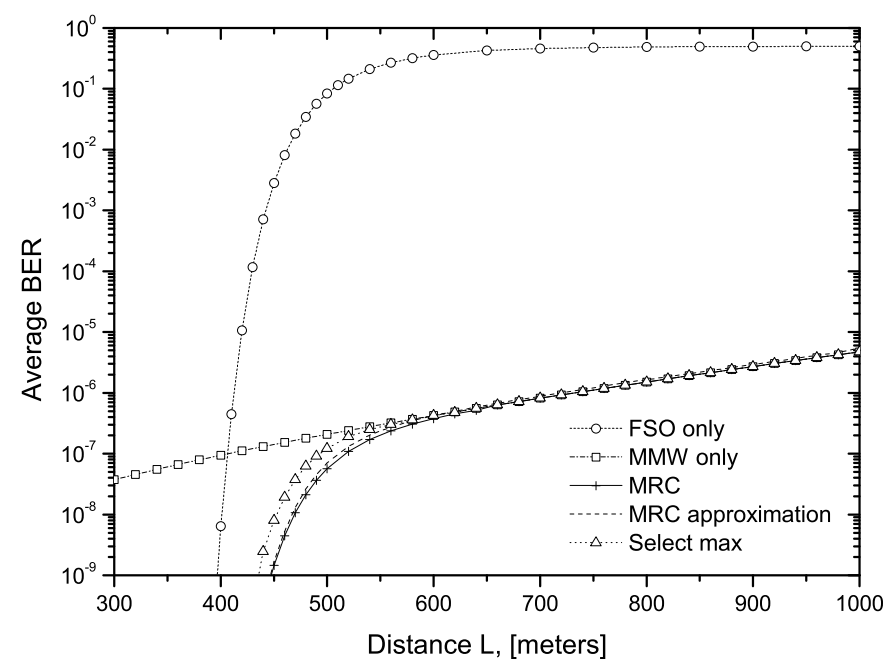

Fig. 3. BER performance of the hybrid RF/FSO system under consideration in moderate fog conditions.

(transmission rate $R_{b}=500 \mathrm{Mbps}$ ) and under clear, moderate rain and moderate fog weather conditions, respectively. In each figure, analytical results for the BER performance of the SC and MRC receiver, derived from (27) and (32) respectively, are plotted as a function of the link distance along with the exact BER performance of the MRC receiver obtained by the numerical integration of (43). For comparison, we also show results when only one of the two links is available at the receiver. It is obvious from the figures that the employment of the combining methods efficiently exploits the complementary nature of RF and FSO links; the performance of both combiners is identical with the performance of the most reliable link, when the other link is totally unreliable, while significant performance gains are offered, when the unreliable link starts to improve. Furthermore, the comparison of the combining methods showed that MRC performs efficiently at higher link distances compared to SC for a certain target BER (for instance, MRC receiver achieves a target BER of $10^{-8}$ at 100 meters further than the range of a SC receiver, under clear weather conditions, while this distance for the same target BER is 10 and 50 meters under moderate fog and moderate rain weather conditions, repectively), which was expected since MRC is the optimum combining method that maximizes the electrical SNR of the output signal. However, this increase at the link distance comes at the cost of complexity, since MRC processes the received signals of both links, in contrast with SC that processes only the link with the highest electrical SNR. Finally, the analytical results for the error performance of the MRC receiver obtained by (32), are very close to the results obtained from the numerical integration of (43) and, thus, the derived analytical expression can be considered as a reliable alternative to cumbersome numerical integration techniques.

\section{Conclusions}

In this paper, we have presented a novel architecture for hybrid RF/FSO wireless systems without feedback or CSI at the transmitter. The described architecture was implemented by transmitting the same data with PSK modulation over both available links, thus enabling the direct combining of the FSO and RF signals at the receiver on a symbol-bysymbol basis. SC and MRC methods were investigated as combining mechanisms and analytical approximations for their BER performance were obtained. Numerical results clearly showed that the proposed hybrid RF/FSO implementation efficiently exploits the complementary nature of FSO and RF links in all weather conditions. Furthermore, the comparison of the diversity combining methods illustrated MRC as the optimum combining method.

\section{APPENDIX I}

In this appendix, we provide details on the derivation of (27). Using (26), (25) can be rewritten as

$$
P_{S C}(e)=I-J
$$

where

$$
I=\bar{P}_{1}(e)
$$

and

$$
\begin{aligned}
J= & \frac{A}{\sqrt{2 \pi}} \int_{0}^{\infty} \exp \left(-\frac{x^{2}}{2}\right) F_{\gamma_{1}}\left(\frac{x^{2}}{2 B^{2}}\right) \\
& \times Q_{1}\left(\sqrt{2 K}, \sqrt{\frac{(1+K)}{\bar{\gamma}_{2}}} \frac{x}{B}\right) d x .
\end{aligned}
$$

The second term of (34) can be evaluated using the Marcum $Q_{1}$-function infinite series representation [18, Eq. (4)], i.e.,

$$
Q_{1}(x, y)=\exp \left(-\frac{x^{2}+y^{2}}{2}\right) \sum_{i=0}^{N} \sum_{j=0}^{i} \frac{x^{2 i} y^{2 j}}{2^{i+j} i ! j !}+\varepsilon(N)
$$

where $\varepsilon(N)$ is a truncation error upper bounded by

$$
|\varepsilon(N)| \leq 1-\frac{\Gamma\left(N+1, \frac{x^{2}}{2}\right)}{N !} .
$$


This upper bound can be derived by rewriting (37), using [13, Eq. $(8.352 / 2)]$, as

$$
\begin{aligned}
Q_{1}(x, y) & =\exp \left(-\frac{x^{2}}{2}\right) \sum_{i=0}^{N} \frac{x^{2 i}}{2^{i} i !^{2}} \Gamma\left(i+1, \frac{y^{2}}{2}\right) \\
& +\exp \left(-\frac{x^{2}}{2}\right) \sum_{i=N+1}^{\infty} \frac{x^{2 i}}{2^{i} i !^{2}} \Gamma\left(i+1, \frac{y^{2}}{2}\right)
\end{aligned}
$$

and since

$$
\lim _{i \rightarrow \infty} \frac{\Gamma\left(i+1, \frac{y^{2}}{2}\right)}{i !}=1
$$

the truncation error of (39) is upper bounded by

$$
\varepsilon(N) \leq \exp \left(-\frac{x^{2}}{2}\right) \sum_{i=N+1}^{\infty} \frac{x^{2 i}}{2^{i} i !^{2}},
$$

which according to [13, Eq. (8.352/2)] is equivalent to (38). Hence, (36) can be rewritten as

$$
\begin{aligned}
J= & \frac{A \exp \{-K\}}{\sqrt{2 \pi} \Gamma(\alpha) \Gamma(\beta)} \\
& \times \sum_{i=0}^{N} \sum_{j=0}^{i} \frac{K^{i}(1+K)^{j}}{\bar{\gamma}_{2}^{j} B^{2 j} 2^{j} i ! j !} \int_{0}^{\infty} x^{2(j-1)} \exp \left(-\frac{x^{2}}{2 q^{2}}\right) \\
& \times G_{1,3}^{2,1}\left[\frac{\alpha \beta x}{\sqrt{2 \bar{\gamma}_{1} B}} \mid \begin{array}{c}
\alpha, \beta, 0
\end{array}\right] d x+\varepsilon_{J}(N)
\end{aligned}
$$

where $\varepsilon_{J}(N)$ is the truncation error for $J$, easily derived to be upper bounded by (29). By expressing the exponent term in the integral of (42) in terms of Meijer's $G$-function, according to [16, Eq. (8.4.3.1)] and using [16, Eq. (2.24.1/1)], the closedform solution of (28) is yielded. This concludes the proof.

\section{APPENDIX II}

In this appendix, we provide details on the derivation of (32). According to (31), the average BER of the MRC combining scheme is given by

$$
\begin{aligned}
\bar{P}_{M R C}= & A \int_{0}^{\infty} \int_{0}^{\infty} Q\left(\sqrt{2\left(\gamma_{1}+\gamma_{2}\right)} B\right) \\
& \times f_{\gamma_{1}}\left(\gamma_{1}\right) f_{\gamma_{2}}\left(\gamma_{2}\right) d \gamma_{1} d \gamma_{2} .
\end{aligned}
$$

The integral in (43) is very difficult, if not impossible, to be evaluated in closed-form. For that reason, we use the approximation for the $Q$-function presented in [19] and, thus the average BER is calculated as

$$
\begin{aligned}
\bar{P}_{M R C} \approx & \frac{A}{12} M_{1}\left(B^{2}\right) M_{2}\left(B^{2}\right) \\
& +\frac{A}{4} M_{1}\left(\frac{4 B^{2}}{3}\right) M_{2}\left(\frac{4 B^{2}}{3}\right)
\end{aligned}
$$

where $M_{1}(\cdot)$ is the moment generating function (MGF) of the Gamma-Gamma channel fading model,obtained in terms of Meijer's $G$-functions, according to [16, Eq. (8.4.3/1)], [16, Eq. (8.4.23/1)] and [16, Eq. (2.24.1/1)], as

$$
M_{1}(x)=\frac{2^{\alpha+\beta-2}}{\Gamma(\alpha) \Gamma(\beta) \pi} G_{1,4}^{4,1}\left[\frac{(\alpha \beta)^{2}}{16 \bar{\gamma}_{1} x} \mid \frac{\alpha}{2}, \frac{\alpha+1}{2}, \frac{\beta}{2}, \frac{\beta+1}{2}\right]
$$

and $M_{2}(\cdot)$ is the MGF of the Rice channel fading model, obtained by [10, Eq. (5.11)], as

$$
M_{2}(x)=\frac{1+K}{1+K+x \bar{\gamma}_{2}} \exp \left(-\frac{K x \bar{\gamma}_{2}}{1+K+x \bar{\gamma}_{2}}\right) \text {. }
$$

By combining (45) with (46) and (44), the analytical expressions of (32) and (33) are obtained. This concludes the proof.

\section{REFERENCES}

[1] L. Andrews, R. L. Philips, and C. Y. Hopen, Laser Beam Scintillation with Applications. SPIE Press, 2001.

[2] F. Giannetti, M. Luise, and R. Reggiannini, "Mobile and personal communications in the $60 \mathrm{GHz}$ band: A survey," Wireless Pers. Commun., vol. 10, pp. 207-243, Jul. 1999.

[3] F. Nadeem, V. Kvicera, M. Awan, E. Leitgeb, S. Muhammad, and G. Kandus, "Weather effects on hybrid FSO/RF communication link," IEEE J. Sel. Areas Commun., vol. 27, no. 9, pp. 1687-1697, Dec. 2009.

[4] H. Wu and M. Kavehrad, "Availability evaluation of ground-to-air hybrid FSO/RF links," Int. J. of Wir. Inf. Networks, vol. 14, no. 1, pp. 33-45, Mar. 2007.

[5] S. Vangala and H. Pishro-Nik, "A highly reliable FSO/RF communication system using efficient codes," in Proc. IEEE Global Telecomm. Conf. (Globecom), Washigton, DC, 2007, pp. 2232-2236.

[6] B. He and R. Schober, "Bit-interleaved coded modulation for hybrid RF/FSO systems," IEEE Trans. Commun., vol. 57, no. 12, pp. 37533763, 2009.

[7] A. AbdulHussein, A. Oka, T. T. Nguyen, and L. Lampe, "Rateless coding for hybrid free-space optical and radio frequency communication," IEEE Trans. Wireless Commun., vol. 9, no. 3, pp. 907-913, 2010.

[8] W. Zhang, S. Hranilovic, and C. Shi, "Soft-switching hybrid FSO/RF links using short-length Raptor-codes: Design and implementation,' IEEE J. Sel. Areas Commun., vol. 27, no. 9, pp. 1698-1708, 2009.

[9] S. Bloom and D. J. T. Heatley, "The last mile solution: Hybrid FSO radio," white paper, AirFiber Inc., 802-0008-000 M-A1, pp. 1-20, May 2002.

[10] M. K. Simon and M.-S. Alouini, Digital communications over Fading Channels. Wiley Interscience, 2005.

[11] S. O. Tatu and E. Moldovan, "V-band multiport heterodyne receiver for high-speed communication systems," EURASIP J. Wireless Commun. and Networking, vol. 2007, no. 34358, Jan. 2007.

[12] J. Li, J. Q. Liu, and D. P. Taylor, "Optical communication using subcarrier PSK intensity modulation through atmospheric turbulence channels," IEEE Trans. Commun., vol. 55, no. 8, pp. 1598-1606, 2007.

[13] I. S. Gradshteyn and I. M. Ryzhik, Table of Integrals, Series, and Products, 6th ed. New York: Academic, 2000.

[14] A. K. Majumdar, "Free-space laser communication performance in the atmospheric channel," Journal of Optical and Fiber Communications Research, vol. 2, no. 4, pp. 345-396, Oct. 2005.

[15] J. G. Proakis, Digital Communications, 4th ed. New York: Mc Graw Hill, 2000.

[16] A. P. Prudnikov, Y. A. Brychkov, and O. I. Marichev, Integral and Series. Vol. 3: More special functions. Amsterdam: Gordon and Breach Science Publishers, 1986.

[17] A. H. Nuttall, "Some integrals involving the Q-function," Naval Underwater Systems Center (NUSC), Technical Report 4297, Apr. 1972.

[18] G. Dillard, "Recursive computation of the generalized Q - function," IEEE Trans. on Aerosp. Electron. Syst., vol. AES-9, pp. 614-615, 1973.

[19] M. Chiani, D. Darbari, and M. K. Simon, "New exponential bounds and approximations for the computation of error probability in fading channels," IEEE Trans. Wireless Commun., vol. 2, no. 4, pp. 840-845, Jul. 2003. 